\title{
Exponetial fitting techniques for the solution of stiff problems with explicit methods
}

\author{
J.I. Montijano*, L. Rández*, M. Van Daele ${ }^{\dagger}$ and M. Calvo* \\ *IUMA - Universidad de Zaragoza \\ ${ }^{\dagger}$ Ghent University
}

\begin{abstract}
In this talk the use of exponentially fitting techniques to solve, by means of explicit RK methods, stiff problems is analyzed. The construction of explicit methods with a stability region adequate for problems in which the spectrum has a gap is studied. The order stiff of the proposed methods is also considered, obtaining conditions that guarantee a prefixed stiff order. Numerical experiments showing the performance of the methods are presented.
\end{abstract}

Keywords: Stiff systems, Exponential fitting, Runge-Kutta, Stability

PACS: 65L05

\section{INTRODUCTION}

Let us consider a constant coefficient, stiff linear system

$$
y^{\prime}=M y
$$

with a spectrum $\operatorname{spec}(M)=\left\{\lambda_{1}, \ldots, \lambda_{m}\right\}, \operatorname{Re} \lambda_{i} \leq 0$. Let us suppose that we integrate this problem with a RK with stability function $R(z)$. If the matrix $M$ can be diagonalized, $M=P \Lambda P^{-1}, \Lambda=\operatorname{diag}\left(\lambda_{1}, \ldots, \lambda_{m}\right)$, the numerical solution $y_{n+1}=R(h M) y_{n}$ satisfies

$$
y_{n+1}=P \operatorname{diag}\left(R\left(h \lambda_{1}\right), \ldots, R\left(h \lambda_{m}\right)\right) P^{-1} y_{n} .
$$

According to this, to have stability the method must satisfy $R\left(h \lambda_{i}\right) \mid \leq 1$ for all the eigenvalues, and for general stiff spectrums A-stability is required and consequently, the RK method must be implicit.

For particular classes of stiff problems, however, there can be explicit methods that can be suitable if they have a stability region adequate to the spectrum of the problem. For example, semidiscretization of parabolic PDEs are known to have negative real spectrum and in this case Chebyshev RK methods [6] can be adequate. These methods are explicit, have order one, and with $s$ stages they have a stability interval $[-\beta(s), 0]$ with $\beta(s) \leq 2 s^{2}$. A practical value of this coefficient is $\beta(s) \simeq 0.653 s^{2}$. Then, you can solve the stiff problem with an explicit RK method but at the price of having low order and probably a large number of (explicit) stages. Thus, for example, if $\lambda_{1}=-10000$ and you want to integrate this problem with a step size of $h=0.1$, you would need at least $s=40$ stages per step.

David Ketcheson [5] has shown that it is possible to develop explicit RK methods whose stability region fits to a prefixed spectrum. In particular, he considered the case when the eigenvalues are located around a value $\lambda_{1} \ll-1$ real and the other eigenvalues are located near $\lambda=0$, with moderate modulus. In other words, problems with gap in their eigenvalue spectrum [2]. The methods obtained in this way have a fixed stability region, and therefore the stepsize $h$ must have a fixed value (or close to it) such that $h \lambda_{i}$ remains in the stability region. If the stepsize must change, due to the behaviour of other components (non stiff for example) of the problem, the method can become unstable.

In this talk, it is shown that the use of exponentially fitting techniques can be useful for certain classes of stiff problems for which the eigenvalue spectrum is located around a few points of the complex plane, such as problems with a gap in their eigenvalue spectrum.

\section{EXPLICIT RK METHODS WITH STABILITY REGION WITH A GAP}

Let us consider a stiff linear system (1) with $\lambda_{1} \ll-1$ real and $\left|\lambda_{j}\right|$ moderate for $j=2, \ldots, m$. Since the stiff character of the solution is due to the component $\exp \left(\lambda_{1} t\right)$, one can think about an exponentially fitted method for which this 
component is integrated exactly. We can consider then a $v$-EF method $[3,4]$ of order $p$ with $v=h \lambda_{1}$ whose stability function will have the form

$$
R(z ; v)=a_{0}(v)+a_{1}(v) z+\cdots+a_{s}(v) z^{s}
$$

where the coefficients $a_{j}(v)$ are functions of the fitting parameter and tend to the coefficients of the underlying nonfitted RK method when $v$ tends to zero. Obviously, $R(v, v)=\exp (v)$ for all step size $h$.

If we apply this method to the linear system (1),we will get

$$
y_{n+1}=P \operatorname{diag}\left(R\left(h \lambda_{1} ; v\right), \ldots, R\left(h \lambda_{m} ; v\right)\right) P^{-1} y_{n}
$$

and the method will be stable whenever the stepsize $h$ is taken small enough to have $\left|R\left(h \lambda_{j} ; v\right)\right| \leq 1$ for $j=2, \ldots, m$.

What happens with the accuracy? The error in the numerical solution is determined by the differences $R\left(h \lambda_{j} ; v\right)-$ $\exp \left(h \lambda_{j}\right)$. By construction this error is zero for $\lambda_{1}$, but for the other eigenvalues we can not guarantee that the error is small for reasonably small step sizes. Note that $R(z ; v)$ tends to the stability function of the underlying RK method when $v \rightarrow 0$, and since the method has order $p$, the fitted method has also order $p$, but this is only valid for small values of $v$, and since $\left|\lambda_{1}\right|$ is very large, $h$ mus be taken very small. In conclusion, with this method the problem can be solved in a stable way for reasonable small step sizes, but to get small errors, we need again to take $h$ in such a way that $|v|=\left|h \lambda_{1}\right|<1$ which requires very small values of $h$.

One can think about the possibility of having a $v$-EF method such that $a_{0}(v)=1, a_{1}(v)=1$ for example, so that the method behaves as a first order method for any value of the fitting parameter $v$. Unfortunately, this is not possible for EF-RK methods, but we can use the same techniques to obtain explicit RK methods that are able to integrate properly the class of stiff problems we are considering. The idea is to build methods that are exact for the problem $y^{\prime}=\lambda_{1} y$, but not necessarily for any problem whose solution is $e^{\lambda_{1} t}$.

Let us start with a first order method. We are searching for a RK method whose stability function $R(z ; v)$ has the form (2) and has order one for any value of $v$. It must satisfy

$$
R(v ; v)=\exp (v), \quad a_{0}(v)=1, \quad a_{1}(v)=1 .
$$

Then, the coefficients of the method must be determined from the conditions

$$
b(v)^{T} e=1, \quad b(v)^{T} c(v)=a_{2}(v), \ldots, \quad b(v)^{T} A(v)^{s-2} c(v)=a_{s}(v):
$$

Let us for example search for a method with order 1 and two stages, $s=2$. The stability function

$$
R(z ; v)=1+z+a_{2}(v) z^{2}
$$

must satisfy $R(v ; v)=\exp (v)$ for all $v$ which implies that

$$
a_{2}(v)=\frac{-1-v+e^{v}}{v^{2}}
$$

The coefficients of the method must then satisfy

$$
b_{1}+b_{2}=1, \quad b_{2} c_{2}=a_{2}(v)
$$

which gives

$$
b_{1}=\frac{1-e^{v}+v+c_{2} v^{2}}{c_{2} v^{2}}, \quad b_{2}=\frac{-1-v+e^{v}}{c_{2} v^{2}}
$$

We have a one-parameter family of methods with Butcher tableau

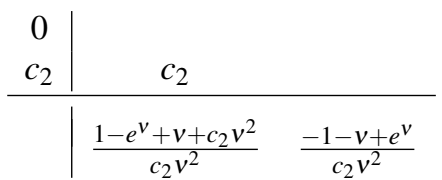

In Figure 1 we plot the values $(x, v), x$ real, where $|R(x ; v)|=1$. We see that until $v \simeq-7$ the interval where there is stability is connected, but for large values of $v$ there is stability for values of $x$ in $[-2,0]$ or around $v$.

The stability region (that does not depend on the parameter $c_{2}$, but it does depend on $v$ ) of the above method is plotted, for $v=-100$, in Figure 2. The right picture shows the region near the origin, and the left one shows the region around $z=v$.

Other methods with higher order have been obtained following this approach. To this end, we have studied the stiff order, obtaining the additional conditions that the coefficients of the methods must satisfy. 


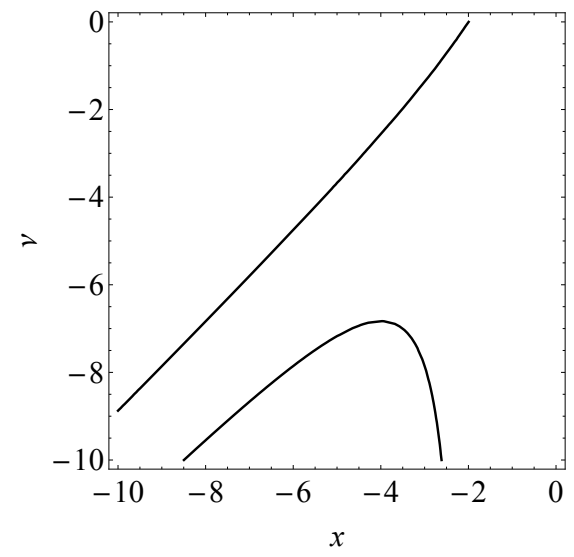

FIGURE 1. Stability interval of the method versus the parameter $v$
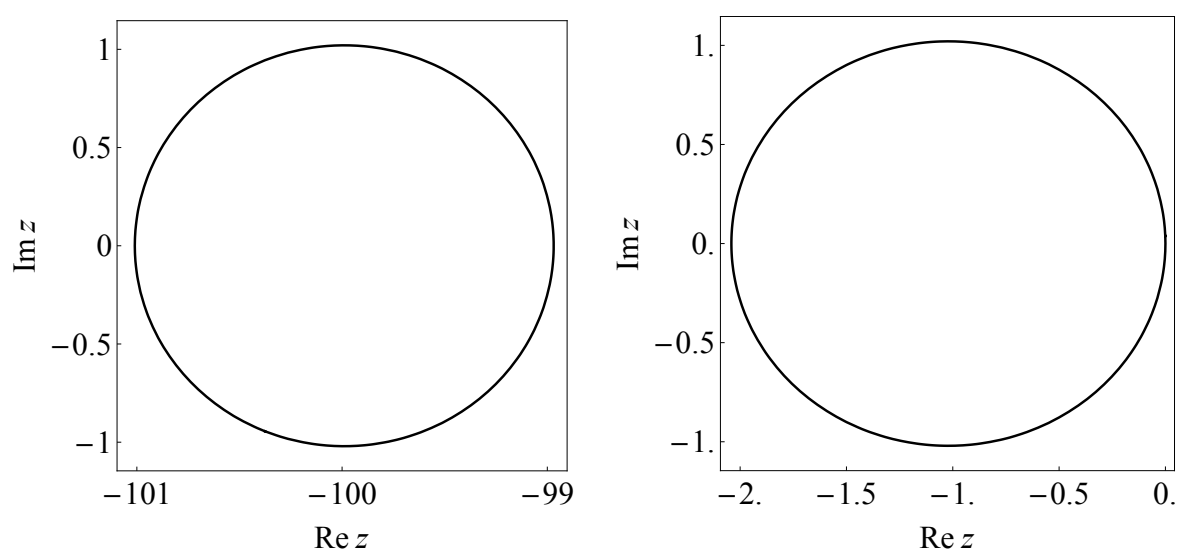

FIGURE 2. Stability region of the method for $v=-100$ around $z=v$ (left) and around $z=0$ (right)

\section{NUMERICAL EXPERIMENTS}

To test the performance of the proposed technique we have integrated some initial value problems with the new obtained methods. We consider here the results obtained with the following linear system whose matrix $M$ has eigenvalues $\lambda_{1}=\lambda_{2}=-1000, \lambda_{3}=-1$

$$
\begin{aligned}
& y^{\prime}=M(y-\varphi(t))+\varphi^{\prime}(t), \quad t \in[0,2] \\
& M=\left(\begin{array}{ccc}
-2099 & -1299 & -999 \\
1099 / 3 & -567 & 333 \\
5195 / 3 & 1865 & 665
\end{array}\right) \\
& \varphi(t)=(\sin (w t), \cos (w t), \sin (w t))^{T} \\
& y(0)=(0,1,0)^{T}
\end{aligned}
$$

We present here the results obtained with a fitted second order method that employs four stages (EF). We also present the results with the well known method of order three of Bogacki and Shampine (BS), used in the code ODE23 in Matlab.

We have used fixed step size $h$ for $h=0.1,0.02,0.01, \ldots, 0.001,0.0002$. In Table 1 we collect the norm of the global error provided at each integration with both methods. As it can be seen, the new method gives a numerical order of 
TABLE 1. Performance of the new method (order two) and BS (order three) with the stiff linear system

\begin{tabular}{rrrr}
\hline Step size & Steps & Error EF & Error BS \\
\hline $10^{-1}$ & 20 & $4 \times 10^{-3}$ & Unstable \\
$2 \times 10^{-2}$ & 100 & $1.5 \times 10^{-4}$ & Unstable \\
$10^{-2}$ & 200 & $3.5 \times 10^{-5}$ & Unstable \\
$2 \times 10^{-3}$ & 1000 & $1.1 \times 10^{-6}$ & Unstable \\
$10^{-3}$ & 2000 & $2.2 \times 10^{-7}$ & Unstable \\
$2 \times 10^{-4}$ & 20000 & $2.5 \times 10^{-9}$ & $1.2 \times 10^{-8}$ \\
\hline
\end{tabular}

about 2 and present a stable behaviour for all the values of the step size. The standard explicit BS method is stable only for $h \leq 2 \times 10^{-4}$, as was expected because its stability interval is $[-2,0]$ and $\lambda_{1}=-10^{4}$.

\section{REFERENCES}

1. J. R. Cash, On the design of high order exponentially fitted formulae for the numerical integration of stiff systems. Numer. Math. 36 (1980/81), no. 3, 253-266.

2. C.W. Gear, I. G. Kevrekidis, Projective Methods for stiff Differential Equations: Problems with gaps in their eigenvalue spectrum, SIAM J. Sci. Comput. 24, No. 4, 1091-1106 (2003).

3. D. Hollevoet, M. Van Daele, Exponentially-fitted methods and their stability functions, J. Comput. Appl. Math. 236, no. 16 (2012) 4006âĂŞ4015.

4. L.Gr. Ixaru, Gr. Vanden Berghe, Exponential Fitting (Kluwer Academic Publishers, Dordrecht, 2004).

5. Ketcheson, David I.; Ahmadia, Aron J. Optimal stability polynomials for numerical integration of initial value problems. Commun. Appl. Math. Comput. Sci. 7, no. 2, 247âĂŞ271 (2012). RKC: An explicit solver for parabolic PDEs

6. B.P. Sommeijer, L.F. Shampine, J.G. Verwer, RKC: An explicit solver for parabolic PDEs, J. Comput. Appl. Math. 88 (1997) 315-326. 